Mirai. Estudios Japoneses

ISSN-e: 2531-145X

\title{
¿Qué es un robot? Análisis jurídico comparado de las propuestas japonesas y europeas $^{1}$
}

\author{
Carmen Tirado Robles ${ }^{2}$ ib
}

Resumen. En este trabajo se pretende realizar un estudio comparativo de lo que se entiende por robot desde el punto de vista jurídico en Europa y en Japón. Este debe ser el punto de partida de cualquier trabajo de investigación sobre las propuestas jurídicas que deben presentarse ante la aparición de los robots en el mundo laboral, económico, industrial, sanitario, cultural, etc. y los retos que tal aparición conlleva. Las dos zonas consideradas, Europa y Japón, lo son por tratarse de las más robotizadas del mundo, junto con China y Estados Unidos.

Cualquier posible respuesta jurídica al reto que supone la entrada de los robots en la sociedad actual implica necesariamente un acuerdo en cuanto a lo que debe entenderse por robot, pues así podremos identificar claramente en qué situaciones o a qué objetos/sujetos podremos aplicar estas nuevas normas jurídicas. Hay que considerar que lo que se entiende por robot es diferente dependiendo de la disciplina desde la que nos queramos aproximar, por lo que la aproximación jurídica tendrá en cuenta, lógicamente, unos condicionantes distintos de otras disciplinas. Acompañando a la definición de robot propondremos también diversas clasificaciones que ya han ido surgiendo y comprobaremos su validez en el plano jurídico. De la comparación de los avances que se han ido produciendo en Europa y en Japón podremos extraer conclusiones útiles para analizar el impacto de los robots y su consideración en las distintas ramas del Derecho.

Palabras claves: Europa, Japón, robot, definición, clasificación

\section{[en] What is a robot? Comparative legal analysis of the Japanese and European proposals}

Abstract. This paper intends to carry out a comparative study of what is understood by "robot" from the legal point of view in Europe and in Japan. This should be the point of departure of any research work on the legal proposals that must be submitted to the appearance of robots in the world of work, economic, industrial, health, cultural, etc. and the challenges that such an appearance entail. Europe and Japan, are the two areas considered because they are the most robotic areas in the world, along with China and the United States.

Any possible legal response to the challenge of the entry of robots into today's society necessarily implies an agreement on what should be understood as a robot, as we can clearly identify in which situations or to which objects/subjects we can apply these new legal rules. It is necessary to consider that what is understood by robot is different depending on the discipline from which we want to approach, so the legal approach will logically have conditions to consider that are different from other disciplines. Accompanying the definition of robot, we will also propose various classifications that have already emerged and we will verify its validity at the legal level. From the comparison of the advances that have been produced in Europe and Japan we can draw useful conclusions to analyze the impact of robots and their consideration in the different branches of law.

Keywords: Europe; Japan; robot; definition; classification

Sumario. 1. Introducción; 2. El concepto de robot en Japón:2.1. Inexistencia de una única definición en la normativa industrial; 2.2. Problemas en la búsqueda de una definición para el ámbito del Derecho; 2.3. Los intentos de definición de los juristas japoneses; 3. El concepto de robot en Europa: 3.1. Algunas cuestiones previas, 3.2. Los esfuerzos de las instituciones de la UE, 3.3. La opinión de los expertos en España. Críticas a la construcción europea; 4. Conclusiones; 5. Bibliografía.

Cómo citar: Tirado Robles, C. ¿Qué es un robot? Análisis jurídico comparado de las propuestas japonesas y europeas, en Mirai. Estudios Japoneses, 4, 2020, 35-48.

\section{Introducción}

El intento de definir qué es un robot no es algo nuevo, pero ha sido siempre un concepto muy cambiante a muchos niveles $\mathrm{y}$, por tanto, difícil de definir de manera válida para una generalidad de situaciones. En primer lugar, porque ha ido cambiando en el tiempo, dependiendo la época en la que se intentara esta definición, la consideración de lo que se

Este trabajo es resultado del proyecto RTI2018-097460-B-I00: Entornos laborales robotizados. Estudio de las propuestas japonesas para la sostenibilidad del gasto público y su aplicabilidad en la UE. Agencia Estatal de Investigación, Convocatorias 2018, Proyectos de I+D+i Retos Investigación (Ministerio de Ciencia, Innovación y Universidades).

2 Universidad de Zaragoza. Grupo de investigación Japón; mctirado@unizar.es 
entendía en el pasado y lo que se entiende en el presente por robot ha cambiado, también hemos de tener en cuenta que la tecnología que produce estos ingenios se ha vuelto cada vez más compleja y sofisticada, aunque el punto de partida ha sido siempre la idea de que un robot es una máquina, independientemente del nivel de autonomía, desarrollo y complejidad que vaya adquiriendo. Cambiante es también este concepto si tenemos en cuenta las distintas vías de aproximación posibles y también dependiendo de la disciplina de la que partamos para dicha definición. En efecto, no será lo mismo la consideración que los robots puedan merecer en el mundo industrial que en el mundo sanitario o en el terreno de la defensa, y tampoco podremos usar la misma definición de robot en psicología, en medicina, en derecho o en periodismo.

A este respecto, estamos de acuerdo con la opinión de Bertolini y Palmerini ${ }^{3}$ cuando afirman que las definiciones que intentan ser comunes a varios campos son poco descriptivas de las aplicaciones robóticas reales y en gran medida engañosas, ya que si se quiere discriminar qué tipo de tecnología califica como robot, los criterios ofrecidos inducirían conclusiones erróneas en la mayoría de los casos. Las definiciones ofrecidas por los investigadores son siempre más precisas y se adaptan mejor al campo de interés específico del hablante, sin embargo, el resultado es fragmentario y contradictorio si se considera una definición general, omnicomprensiva y dirigida a múltiples disciplinas.

Con razón advierte Javoleno: Omnis definitio in iure civili periculosa est: parum est enim, ut non subverti posset que puede traducirse afirmando que "toda definición en el Derecho civil es peligrosa, pues es difícil que no pueda ser alterada" y esto puede servirnos en todas las ramas del Derecho y en todas las ciencias, no solo en la jurídica. Aun así, el objeto de este estudio es indagar sobre las diferentes definiciones y clasificaciones de los robots en el Derecho desarrollado en Europa y en Japón.

Se suele citar como punto de partida ${ }^{5}$ el uso del término "robot" por parte del escritor de ciencia ficción, de nacionalidad checoslovaca, Karel Čapek ${ }^{6}$, mientras que la palabra "robótica" se atribuye a Isaac Asimov, también escritor de ciencia ficción, además de profesor de bioquímica en la Facultad de medicina de la Universidad de Boston. Asimov establece las llamadas tres leyes de la robótica como un conjunto de normas que se aplican a la mayoría de los robots de sus novelas y cuentos y que están diseñados para cumplir órdenes ${ }^{7}$. En ese universo, las leyes son "formulaciones matemáticas impresas en los senderos positrónicos del cerebro" de los robots (líneas de código del programa que regula el cumplimiento de las leyes guardado en la memoria principal de aquellos). Aparecidas por primera vez en el relato Círculo vicioso (Runaround, 1942), de la manera que sigue (aunque su forma real sería la de una serie de instrucciones equivalentes y mucho más complejas en el cerebro del robot):

1) Un robot no hará daño a un ser humano o, por inacción, permitirá que un ser humano sufra daño.

2) Un robot debe cumplir las órdenes dadas por los seres humanos, a excepción de aquellas que entrasen en conflicto con la primera ley.

3) Un robot debe proteger su propia existencia en la medida en que esta protección no entre en conflicto con la primera o con la segunda ley.

Así, en una primera aproximación, podemos decir que el mundo de los robots debe mucho a la literatura de ciencia ficción, ya que esta ha sido la manera de hacer este mundo si no comprensible, al menos asumible para el gran público. Es a partir de la década de 1970 cuando la robótica se desarrolla apoyada en el avance de las ciencias informáticas y va ganando en especialización, diversificando sus funciones y desarrollándose las múltiples aplicaciones que un robot puede tener. A partir de ese momento empiezan a surgir diferentes definiciones del término robot y diferentes clasificaciones.

Como ya hemos adelantado, vamos a fijarnos específicamente en las definiciones que desde el ámbito del Derecho se han ido presentando en el ámbito europeo y en Japón pues ambas áreas geográficas están fuertemente robotizadas y estas definiciones quizás puedan hacerse extensivas a toda la comunidad internacional. Japón es, quizás, el país con más alto grado de desarrollo tecnológico del mundo, por lo que el uso de robots está muy extendido, no solo en la industria, sino también en ámbitos como el sanitario, la ayuda doméstica, la asistencia a ancianos y personas con discapacidad, la ingeniería civil e incluso los espectáculos o el entretenimiento. A diferencia de Japón, donde el paro se sitúa en un $3 \%{ }^{8}$, el entorno europeo tiene graves problemas de desempleo y un sistema de pensiones cuyo futuro

Véase Bertolini, A. / Palmerini, E. (2014): 178-179.

El conocido proverbio es expresado así en Digesto, 50.17.202: Iavolenus 11 Epistulae.

Se pueden ver referencias históricas anteriores en García-Prieto Cuesta, J. (2018): 27-60.

El término aparece por primera vez en su obra de teatro R.U.R. (Robots Universales Rossum), en 1920, cuyo éxito primero en Praga, y luego en Londres y Nueva York, hace que el uso del término se extienda a otras lenguas. Etimológicamente deriva de la palabra checa robota "trabajo" y fue empleado por primera vez en esta obra; según el autor italiano Ripellino también tiene relación con el antiguo eslavo rob ( $p ъ \sigma$, 'esclavo'). El término en sí se atribuye a Čapek, pero fue su hermano Josef el que, al estar pintando, según se cuenta, y ser preguntado por Karel con qué palabra le parecía a él que podía designar a los androides trabajadores que tenía en mente, farfulló entre pinceles algo parecido a "robota" (véase Sáiz Lorca, D. (2006): 145-146.

Véase De La Parra Trujillo, E. (2017): 165-188.

Al Japón de hoy en día no le preocupa tanto el desempleo, pues es un problema prácticamente inexistente en el ámbito interno, pero sí tiene varios problemas que son comunes también al ámbito europeo, como indica certeramente Gascón Marcén, A. (2020) señalando que la UE y Japón enfrentan desafíos similares con el envejecimiento de la población, una baja tasa de fertilidad y un costoso estado de bienestar. En este sentido, un campo en el que la cooperación japonesa y de la UE puede ser especialmente útil es la gobernanza de la inteligencia artificial (IA). Ambos actores sienten que están perdiendo la carrera con Estados Unidos y China y, en muchos aspectos, esto es cierto. Japón y la UE han decidido invertir fuertemente en investigación y desarrollo (I + D), la UE a través de su programa Horizon Europe y Japón a través de su programa Moonshot, pero esto, advierte Gascón Marcén, puede no ser suficiente en comparación con sus competidores. 
está en riesgo, por lo que la entrada de los robots en el mundo laboral viene a incrementar estos problemas y puede hasta provocar cierta alarma social.

La mayor parte de los investigadores japoneses coincide en que lo que se destruiría con el uso masivo de robots serían, sobre todo, puestos de cualificación media/baja y trabajos consistentes en tareas monótonas o repetitivas, por lo que sería necesario establecer concretos programas de formación de los empleados que los desempeñaban, al objeto de dotarlos de nuevas capacidades técnicas. Lo cierto es que hay estudios serios e igualmente fiables en ambos sentidos: unos que defienden que habrá una destrucción masiva de puestos de trabajo y otros, más optimistas, que consideran que los empleos destruidos serán compensados por la creación de otros nuevos. La historia nos dice que, en el pasado, el $80 \%$ de la población trabajaba en el campo y las revoluciones industriales lo que han hecho es aumentar el número de trabajadores, por lo que, en principio, no deberíamos temer la pérdida de puestos de trabajo. Lo que está claro es que los ciudadanos están preocupados por este tema y lo prudente es estudiarlo con rigor y seriedad, huir de los tópicos y buscar soluciones reales que puedan contribuir a garantizar una vida digna de los habitantes del planeta. Ahora bien, la transformación del empleo y el hallazgo de nuevos nichos de empleo requerirá su tiempo y lo más probable es que los Estados tengan que enfrentarse a bolsas de desempleo provocadas por la "brecha digital". Entretanto, el sistema educativo y el económico deberán absorber a los desempleados y los Estados se verán forzados a aumentar su gasto en ayudas sociales, mayor financiación del sistema educativo, etc. La búsqueda de nuevas fuentes de ingresos públicos se planteará en el medio plazo. Una de las más reconocibles es la que propugna un gravamen a los robots.

En todo caso, aunque el panorama sea ciertamente distinto en Europa y en Japón, ambas sociedades contemplan la implantación de los robots tanto desde el punto de vista de las oportunidades como desde el punto de vista de los retos, pero en todo caso, a efectos jurídicos es necesario en primer lugar considerar cuál es el fenómeno del que estamos hablando. Así, hay que contestar a la pregunta ¿qué es un robot?

\section{El concepto de robot en Japón?}

\subsection{Inexistencia de una única definición en la normativa industrial}

La sociedad japonesa tiene una gran tradición en la fabricación de autómatas (llamados en japonés karakuri-ningyo). Es famoso el artesano japonés Tanaka Hisashige (1799-1881), conocido como el "Edison japonés", que creó una serie de juguetes mecánicos extremadamente complejos, algunos de los cuales servían té, disparaban flechas sacadas de un carcaj e incluso eran capaces de trazar $k a n j i^{10}$. En Japón también se asume la referencia a la obra de Capek definiendo robot con carácter general como 人の代わりに何らかの作業を自律的に行う装置、もしくは機械の こと, "Máquina o dispositivo que efectúa ciertas labores de modo autónomo en sustitución de los humanos", incluso estas fuentes ${ }^{11}$ indican que los robots se pueden clasificar en dos grupos:

a) Máquinas capaces de realizar trabajos automáticos de modo continuo o aleatorio, con un cierto grado de autonomía, como robots industriales, militares, de limpieza, etc.

b) Máquinas cuya apariencia y funciones pretenden imitar las de personas o animales, lo que incluye también los trajes a modo de exoesqueleto y dispositivos que aumentan la fuerza, entre otros.

También existen definiciones sectoriales específicas, como las que aparecen en varias normas JIS ${ }^{12}$, por ejemplo, la norma JIS B0134 (1979) definía robot como "Sistema mecánico que, dotado de funciones de movimiento flexible análogas a las de los organismos vivos, puede combinarlas con funciones inteligentes y actuar dando respuesta a la voluntad humana". Añade que, en este contexto, por "función inteligente" hay que entender la capacidad para llevar a cabo al menos una de las siguientes tareas: juicio, reconocimiento, adaptación o aprendizaje. Posteriormente, el JIS B0134 (1998) definió al robot industrial como "Máquina utilizada en la industria, dotada de funciones de manipulación o traslado con control automático y capaz de

\footnotetext{
La autora desea agradecer al Dr. Antonio Francisco Barberán Pelegrín, coordinador de lengua japonesa en el Centro de lenguas modernas de la Universidad de Zaragoza y abogado en ejercicio, su trabajo de búsqueda y traducción de las fuentes japonesas.

10 Hornyak, T. N. (2006) se refiere al trabajo de Tanaka especialmente en p. 25.

11 Véase j-net21.smrj.go.jp (2020)

12 La abreviatura JIS hace referencia al Japan Industrial Standards Comittee o Comité de Normas Industriales de Japón [www.jisc.go.jp/eng]. Esta normativa de calidad japonesa no coincidía con la establecida internacionalmente, por lo que, en opinión de Cisneros Cristóbal, P. (2019): pp. 173209, resultaba mucho más complicado comerciar con el país nipón y presentaba problemas a la hora de negociar determinados puntos del reciente Acuerdo de Libre Comercio. Efectivamente, Japón tiene un sistema propio que se basa en la Ley de Estandarización Industrial que se promulgó en junio de 1949 y luego ha ido reformándose con el paso del tiempo. Las JIS sirven para la acreditación y certificación de todos los productos industriales, exceptuando aquellos regulados por leyes nacionales específicas o por otro sistema de normativas (véase información de JETRO (20??). Estas normas equivalen a las normas ISO (International Organization for Standardization), que se ocupa de la elaboración de normas técnicas de carácter voluntario a nivel internacional, normas que se usan en las empresas para una mayor eficiencia y rentabilidad económica, por lo que, gracias a ellas, los intercambios comerciales entre países son más fáciles y justos. Aunque en la mayor parte del comercio internacional es muy habitual el uso de las normas ISO, lo cierto es que Japón tiene sus normas de calidad. A comienzos del siglo XXI Japón ha ido acogiendo el sistema internacional ISO, sobre todo a raíz de los debates sobre la lista de barreras no arancelarias que el comercio con Japón implica superar, así Japón ha iniciado un cambio en sus políticas hacia un mayor uso de normas internacionales, en especial, las que se emplean también en la UE, como el etiquetado de textiles, vehículos de motor o productos farmacéuticos. Véase Introducción al Acuerdo de Asociación económica... (2017).
} 
ejecutar diversos tipos de operaciones mediante su programación”. Finalmente, la norma JIS B0134 (2015) define robot como "Estructura móvil de dos o más ejes que, dotada de un cierto grado de autonomía, opera mediante programación y ejecuta las operaciones requeridas dentro de un determinado entorno"13. Aclara que el robot debe contar también con un sistema de control y una interfaz para éste, y que su clasificación en industrial o de servicio dependerá de su uso.

Centrándonos ya en normas jurídicas que hacen referencia al concepto de robot e intentando una definición, destacamos aquí dos normas de carácter técnico que incluyen definiciones, la primera es el Reglamento de Salud y Seguridad en el Trabajo, en su artículo 36, apartado xxxi que define al robot industrial como "Máquina dotada de dispositivos de manipulación y de memoria (incluidos los dispositivos de control de secuencia variable y los de control de secuencia fija) que, usando la información almacenada en los de memoria, es capaz de realizar, mediante los de manipulación y de modo automático, movimientos de expansión y contracción, flexión y extensión, movimiento o traslación en vertical y en horizontal, giros, y acciones combinadas de todos ellos" ${ }^{4}$, mientras que el Reglamento que desarrolla la Ley de ondas electromagnéticas y radiodifusión define a los robots radio-meteorológicos en su artículo 2, apartado xxxxiii como "equipos de radio destinados a prestar servicios de asistencia a las tareas de meteorología que, instalados en la tierra o en el mar, emiten o retransmiten automáticamente datos meteorológicos"15.

Como máquinas que trabajan en sustitución de los humanos, la definición debería abarcar a las que realizan de forma automática y continua una cierta cantidad de procesos (aunque se trate de dispositivos que solo realizan una operación sencilla, como las cintas transportadoras, las escaleras mecánicas, etc.), pero no incluye ni a las que requieren de un operador humano permanente (como los ascensores), ni a un operador que suba a ellas y las maneje o pilote (como excavadoras y bulldozers).

Sin embargo, si la máquina tiene apariencia humana, siempre se suele incluir en la categoría de robot, aunque su manejo sea completamente operado por un humano de modo manual. Los vehículos o máquinas de trabajo de apariencia humana pilotados (tipo Mazinger Z) o los exoesqueletos motorizados también se consideran robots. Respecto a la cuestión de la apariencia humana de los robots, es conocido el curioso fenómeno del "valle inquietante" (uncanny valley o bukimi no tani en japonés), hipótesis original del robotista japonés Masahiro Mori, profesor del Instituto de Tecnología de Tokio, en una investigación presentada en 1970: Mori ${ }^{16}$ observó que, a medida que aumentaba la semejanza humana de los robots, también aumentaba la percepción positiva, de familiaridad, de los observadores. No obstante, esta correlación positiva se invertía justo cuando los robots alcanzaban un grado de realismo que les dio una apariencia casi humana, pero no perfecta: los observadores calificaban esta percepción como excepcionalmente negativa. Mori trazó esta relación entre el realismo humano, la percepción positiva, de familiaridad y la repentina "inmersión" en un pico negativo en el "nivel casi humano" y la denominó "valle inquietante".

Más difícil resulta determinar hasta qué punto son robots las máquinas dotadas de sistemas de control remoto, autoconducción o piloto automático, pues su calificación como robots parece depender del grado de intervención humana en su manejo. La tecnología ha avanzado tanto que, en muchas de ellas, la intervención humana se limita solo a dar las instrucciones u órdenes que queremos que la máquina cumpla. En este sentido puede decirse que asistimos a una suerte de proceso de robotización de muchas máquinas. Los dispositivos asistentes de manipulación capaces de transmitir mecánica o eléctricamente las acciones humanas, como los robots quirúrgicos o los brazosrobot, aunque se denominan así, no serían, entonces, estrictamente robots.

Por el contrario, aunque sean entes incorporales, algunos programas de software sí podrían encajar en la categoría de "entes que trabajan en sustitución de humanos realizando determinados procesos de modo automático y continuo", como los motores de búsqueda o los programas que buscan automáticamente información por Internet (búsqueda mediante bots). De hecho, se les llama bots para distinguirlos de los robots mecánicos.

Por tanto, tras lo dicho podemos concluir que en las normas de carácter industrial no hay una única definición de robot, aunque sí se pueden encontrar algunos elementos que podrían conformar esta definición, ya fueran en concurrencia o separadamente: el primer elemento es el hecho de que el robot se crea para sustituir el trabajo humano, por lo que, en segundo lugar, el robot debería imitar en alguna medida el comportamiento humano. De estas definiciones, en principio, no cabe deducir que el robot deba tener apariencia humana, aunque este elemento pueda ser determinante en algunos ámbitos para incluir al ingenio en esta categoría. El último requisito necesario sería la actuación de modo autónomo.

\subsection{Problemas en la búsqueda de una definición para el ámbito del Derecho}

En este apartado vamos a hacer referencia a los problemas que han surgido en Japón cuando los juristas han intentado encontrar una definición de robot $\mathrm{y}$, a nuestro modo de ver, son tres principalmente: el solapamiento con otros

\footnotetext{
Véase la última versión de esta norma en kikakurui.com (2012).

Véase Ordinance on Industrial Safety...(1972).

Véase Regulations for Enforcement of the Radio... (1950).

Véase Mori, M. (2012), traducción de Karl F. MacDorman and Norri Kageki autorizada por el autor. La representación en un gráfico se realiza como una línea ascendente, reflejando la percepción positiva, seguida de una línea descendente reflejando la percepción negativa, similar al dibujo de una montaña y un valle, de ahí el nombre elegido por Mori. Esta teoría del valle inquietante ha sido seguida y estudiada por muchos robotistas en todo el mundo, véase, entre otros, Esteve, F. (2015), también Wong, S. (2017), y, más recientemente, Venanzoni, A. (2019): 237-280.
} 
conceptos, la obsolescencia de las definiciones y la inadecuación de los términos usados por otras disciplinas para su uso en el ámbito jurídico.

Efectivamente, en primer lugar, hay que señalar la existencia de términos cercanos, relacionados o conexos con el de robot, lo que hace que la búsqueda de una única definición en el ámbito jurídico se haga más difícil y también su deslinde de esos otros conceptos. Nos referimos a términos como los de persona electrónica, inteligencia artificial, dron, ciborg y otros.

Así, los términos IA y robot se solapan actualmente en Japón, lo que se percibe claramente en el informe del "Grupo de Investigación de Medidas en relación con los Robots" del Ministerio de Economía, Comercio e Industria de $2006^{17}$, que define robot como máquina dotada de:

\section{1-Sensores}

2-Inteligencia o sistema de control

3-Sistema motriz

Pero lo cierto es que paulatinamente, con el desarrollo de la ciencia, la necesidad de sensores o de sistema motriz ha pasado a segundo plano y deja de ser indispensable contar con esos elementos, por lo que la definición se centra en la inteligencia o sistema de control, lo cual acaba equiparando a menudo a un robot con una IA. Dicho de otro modo, el robot ha pasado a ser una "máquina inteligente" y ello dificulta su diferenciación con la IA. Veremos que también en el entorno europeo se da una cierta asimilación, aunque realmente sean conceptos claramente diferentes en el plano teórico, como vamos a ver después.

Esto nos lleva a tener en cuenta una circunstancia que se da especialmente cuando nos enfrentamos a cuestiones relacionadas con el desarrollo tecnológico, como es esta cuestión de los robots, y es el hecho de la obsolescencia del término, con lo que volveríamos a la idea de Javoleno: la ciencia avanza mucho más rápido que el Derecho y, añadimos, los conceptos que crea el Derecho devienen obsoletos con el paso del tiempo pues los cambios jurídicos son lentos.

Finalmente, como hemos visto, un tercer problema es que las normas referidas al ámbito industrial han ensayado ciertas definiciones de robot que no sirven a efectos jurídicos. Algunos autores, como los profesores Yanaga ${ }^{18}$ o Shishido ${ }^{19}$, consideran que las definiciones de las normas JIS, propias del ámbito de la ingeniería, no resultan adecuadas para definir lo que es un robot en el ámbito jurídico y en la época actual. Es más, aún se puede añadir un problema más si consideramos en exclusiva al mundo del Derecho, y es que, por existir diversas disciplinas jurídicas que contemplan problemas muy diferentes, puede suceder que la definición de robot dada para el ámbito penal no sea válida para los asuntos laborales o fiscales, y que esta no sea válida para los problemas éticos que contempla la filosofía del Derecho o no sirva para dar solución a los problemas de responsabilidad por daños que se estudian en el Derecho civil, y así sucesivamente.

Como conclusión, debemos preguntarnos si será posible no solo encontrar una definición de robot para el mundo del Derecho, sino si esta definición podrá servir para todas las ramas del Derecho en las que se plantean problemas derivados de la implantación de los robots en la sociedad.

\subsection{Los intentos de definición de los juristas japoneses}

Lo cierto es que cuando se introducen robots en una sociedad, como la japonesa, se requiere que estos robots se ajusten a las leyes y regulaciones existentes y, en el caso de que tales leyes y regulaciones se consideren inapropiadas teniendo en cuenta las circunstancias socio-económicas, será necesario introducir enmiendas a estas leyes. Esto es lo que ha pretendido la Japan Robot Association (JARA), heredera de la antigua Japan Industrial Robot Association (JIRA), que adopta una Nueva Estrategia sobre los robots, presentada en 2015 por el Ministerio de Economía, Comercio e Industria ${ }^{20}$ en la que hay un apartado dedicado específicamente a la necesidad de cambios legislativos a causa de la irrupción de los robots en el entorno social y económico japonés.

Esta Nueva Estrategia parte de la idea de que, para promover y ampliar la utilización de robots en la sociedad real, se debe llevar a cabo una reforma equilibrada de las regulaciones e instituciones desde el punto de vista tanto de la desregulación como del establecimiento de nuevas y adecuadas reglas. Así, se pretende tomar medidas para establecer nuevas reglas para la colaboración de humanos y robots basadas en una comprensión profunda de la realidad sobre los robots y con la colaboración del Consejo de Reforma Reguladora, adscrito al Gobierno, al tiempo que se eliminan las regulaciones innecesarias.

El documento tiene en cuenta el período 2015-2020 y señala la necesidad de reformar la regulación con respecto a la utilización del robot marcando dos prioridades en el campo jurídico: la primera, la desregulación y el establecimiento de un nuevo sistema legal para hacer un uso efectivo de los robots y, en segundo lugar, el establecimiento del marco requerido desde el punto de vista de los consumidores.

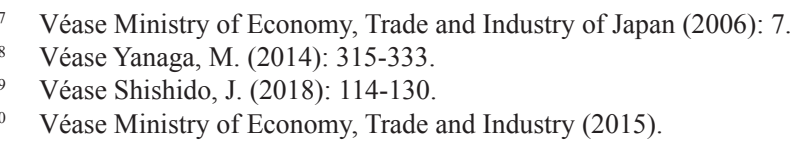


Las cuestiones a tratar concretamente y los planes de acción se organizan de la siguiente manera: en primer lugar, se tiene en cuenta el criterio de la reciprocidad a nivel internacional, por lo que la aplicación de nuevas regulaciones se hará bajo el supuesto de que se aplican recíprocamente tanto en el país como en el extranjero. En segundo lugar, se pone en marcha la "Iniciativa Revolución de los robots"21 que juega un papel central en esta reforma, siempre en armonía con la investigación y el desarrollo de tecnología de hardware y software de robots.

1) Desregulación y establecimiento de un nuevo sistema legal para hacer un uso efectivo de los robots: las reformas se centran en la modificación de ciertas leyes, y se citan algunos ejemplos específicos que requerirán estos cambios, como una legislación que establezca un nuevo sistema de utilización de ondas de radio que admita la utilización de robots, la Ley sobre calidad, eficacia y seguridad de productos farmacéuticos, dispositivos médicos, productos de terapia regenerativa y celular, productos de terapia genética y cosméticos que tenga en cuenta a los robots, un sistema de seguros médicos a largo plazo, nuevas leyes de tráfico por carretera y de vehículos de transporte por carretera, Leyes y reglamentos relacionados con los robots de tipo aéreo no tripulados (Ley de aviación y similares), Leyes y reglamentos relacionados con el mantenimiento y reparación de infraestructuras públicas o una nueva Ley de seguridad de gas a alta presión.

2) Establecimiento del marco requerido desde el punto de vista de los consumidores: las reformas afectan a leyes como la de seguridad de productos de consumo, la Ley de electrodomésticos y la de seguridad de materiales. Si suponemos que vamos a vivir en una sociedad en la que los robots lleguen a todos los rincones de la vida diaria y se amplíe la utilización de los robots de próxima generación relacionados con la vida cotidiana, que tienen autonomía y capacidad de control remoto, es necesario fortalecer las medidas para garantizar la seguridad del consumidor, también habrá que tener en cuenta la recopilación de información y la investigación de la causa cuando se produce un accidente grave del producto debido a robots, las normas técnicas sobre equipos tratados como aparatos eléctricos que pueden considerarse robots o el nivel de responsabilidad de los fabricantes y similares, según las tendencias de desarrollo tecnológico y comercialización específica. Hay que asumir, según los expertos japoneses que elaboran este documento, que en el sistema jurídico actual todavía no se asume la utilización del robot.

Señala este documento que el concepto de robot debe ampliarse para incluir los nuevos avances tecnológicos y el potencial de desarrollo futuro con un enfoque flexible de lo que entendemos por robot.

En contraste con los puntos de vista convencionales que suelen tener en cuenta a los robots que hemos mencionado más arriba, máquinas equipadas con tres elementos (sensor, inteligencia/control y sistema motriz), existe una nueva estructura emergente construida sobre el avance de la digitalización, el enriquecimiento de la computación en la nube y el progreso de IA, donde los robots pueden ser manejados por un control independiente basado en IA que permite el acceso a varias personas y objetos en el mundo real sin la ayuda de un sistema de actuación específico. Por ello, la definición convencional de robots ya no puede ser la misma.

Por otra parte, algunos juristas japoneses han intentado dar una definición de robot, por ejemplo, el profesor Hirano $^{22}$, lo define como "Una máquina dotada de ciclos de detección o reconocimiento, con pensamiento o juicio y acción” (感知・認知）＋（考え・判断）＋（行動）の循環を有する機械.) Otros, como Asami ${ }^{23}$ distinguen dos tipos de robots, los industriales y los de servicio, considerando que el "robot de servicio" es un término genérico que abarca todos los robots que no son para uso industrial, es decir, que no están destinados a racionalizar la producción en un sitio de fabricación. Se pueden concebir también dos clases de robots integrados en el término robots de servicio, según el mismo autor:

Clase A. Robots para reemplazar a los seres humanos en el trabajo en operaciones sucias, peligrosas y/o tediosas, en donde este autor incluye trabajos en entornos difíciles (como altas temperaturas, vacío, bajo el agua, entornos radiactivos), lucha contra incendios, aplicaciones policiales y militares, trabajos de construcción, publicidad, entretenimiento y tareas domésticas repetitivas.

Clase B. Robots para operar en o con seres humanos con el fin de aliviar la incomodidad o aumentar el confort. Aquí se incluirían los robots usados en medicina para la atención al paciente, los guía para ciegos, los robots usados en casas de juego o en parques de atracciones y algunos usados para tareas domésticas como el servicio de mesa.

Según este autor, las operaciones o servicios que se realizarán en las actividades anteriores podrían ser adecuadas en algunos casos para robots que presentan una apariencia similar a los seres humanos, pero mucho más a menudo, los robots asumirían una forma completamente original para que coincida con la función específica para la que están destinados.

\footnotetext{
Esta iniciativa se establece como un canal central de colaboración a distintos niveles para avanzar con el progreso material. La Robot Revolution Initiative emprenderá tareas tales como 1) la promoción de la colaboración público-privada y la correspondencia entre usuarios y fabricantes, así como la recopilación y difusión de información relevante, 2) la planificación y redacción de proyectos de investigación conjunta internacional como el desarrollo conjunto de robots para la prevención de desastres por parte de Japón y Estados Unidos, 3) la elaboración estratégica y utilización de estándares globales, propuestas de reformas regulatorias y establecimiento de reglas sobre seguridad de datos, y 4) el intercambio y divulgación de las mejores prácticas.

22 Véase Hirano, S. (2017).

23 Véase Asami, S. (1994): 22-26.
} 
Volviendo otra vez al trabajo de la Japan International Robots Association (JIRA), esta ha clasificado a los robots en seis grupos diferentes, teniendo en cuenta su nivel de inteligencia ${ }^{24}$ :

- Clase 1: Dispositivos de manipulación manual: los maneja un operador acreditado y tienen distintos grados de libertad.

- Clase 2: Robot de secuencia fija: utiliza un mecanismo predeterminado fijo para realizar etapas sucesivas de una tarea, por lo que es difícil modificar este funcionamiento.

- Clase 3: Robot de secuencia variable: variante fácil de modificar si lo comparamos con la clase 2.

- Clase 4: Robot de reproducción: la máquina registra los movimientos del operador humano y luego los replica.

- Clase 5: Robot de control numérico: un programa de control de movimiento reemplaza los movimientos grabados de los robots que han sido clasificados en la clase 4.

- Clase 6: Robot inteligente: tiene medios para comprender el entorno y capacidad para completar con éxito una tarea a pesar de los cambios que se produzcan en las condiciones del entorno.

La mayor parte de los robots en Japón pertenecerían a las clases 2 y 4 y, en cambio, para otras asociaciones como la Robotic Industry Association (RIA) de Estados Unidos, las dos primeras clases no estarían incluidas en sus estadísticas como robots pues no son reprogramables, y para la asociación americana no habría distinción entre las otras cuatro clases de robots que sí distingue la asociación nipona. Esto sucede porque a la JIRA no le interesaba la IA aplicada a un robot, dado que su fin era ser manejado por un humano, sin embargo, sí destaca en su definición y clasificación su capacidad de movimiento y su analogía con los seres de la naturaleza; en cambio, y así lo indica Zabala ${ }^{25}$, para la asociación americana lo remarcable es la capacidad de actuación de un robot independientemente de la acción humana.

Finalmente, una definición general que conjuga elementos tecnológicos con elementos jurídicos puede ser la siguiente: "Sistema capaz de captar datos externos, comprender su significado, juzgar por sí solo qué debe hacer y, como resultado de ello, actuar al servicio de los humanos"26. La abogada Nanbu Tomoko, de la corporación RiverCity Horitsu Jimusho ${ }^{27}$, señala el incremento exponencial en la jurisprudencia japonesa de casos en los que se hayan involucrados robots (en su investigación encontró 105 casos en 2005 y 210 referencias en 2010). Por otra parte, la autora propone como mejor solución, dada la situación jurídica actual de los robots en Japón, la de establecer una definición específica para cada campo concreto, del tipo: “A efectos de esta ley, se entenderá por robot...”, opinión que, por ahora, pensamos que es la más acertada.

\section{El concepto de robot en Europa}

\subsection{Algunas cuestiones previas}

$\mathrm{Al}$ igual que sucede en Japón, también en el entorno europeo la escasez de definiciones propias del ámbito jurídico, unida a la proliferación de definiciones en otros ámbitos ${ }^{28}$, su solapamiento y superposición con otros conceptos y su difícil deslinde entre éstos, hace muy difícil aspirar a una definición universal de robot, válida para todos los ámbitos del Derecho. También aquí nos encontramos con diversos puntos de vista, distintas definiciones, así como clasificaciones muy diferentes en el ámbito de la tecnología, pero lo cierto es que parece evidente que las definiciones suelen ser muy restrictivas, pues suelen centrarse en robots de características o finalidades muy determinadas, y, además, se desactualizan rápidamente, obedeciendo a esa idea antes comentada de la evolución rápida de los avances tecnológicos.

La primera dificultad surge de la diferencia conceptual entre el mercado japonés y el euro-americano de lo que es un robot y lo que es un manipulador. Así, mientras que para los japoneses un robot industrial es cualquier dispositivo mecánico dotado de articulaciones móviles destinado a la manipulación, el mercado occidental es más restrictivo, exigiendo una mayor complejidad, sobre todo en lo relativo al control. En segundo lugar, y centrándose ya en el

\footnotetext{
Véase Poole, H. (1989).

En Zabala, G. (2007): pp. 18-19.

26 En Taniwaki, Y., (2018). En el original japonés: 外界のデータを取り込み、その意味を理解して、何をすべきかを判断し、結果として人 に役立つように働きかけるシステムである（岩波書店「岩波講座ロボット学 1 ロボット学創成」5 5 ページ）。

27 Es una corporación de abogados japonesa que ha realizado un informe de investigación bastante completo, titulado Cuestiones legales sobre robots (ロボット法.ロボットに関する法律問題). Véase Nambu, T. (2019).

28 Mercader Uguina, J. (2018), en donde el autor pone algunos ejemplos de definiciones de robot en las normas ISO aplicables en todo el entorno internacional y, por supuesto, en Europa: la ISO 8373:2012 define al robot industrial como un manipulador programable en tres o más ejes multipropósito, controlado automáticamente y reprogramable; las ISO 10218-1: 2012 e ISO 10218-2:2012 que tratan sobre robots colaborativos, que comparten el mismo espacio de trabajo con los humanos; o la nueva especificación técnica ISO/TS 15066:2016 (Robots and robotic devices), que precisa los requisitos de seguridad para los sistemas de robots colaborativos. Véase Robotics. https://www.robotics.org/product-catalog-detail. cfm/Robotic-Industries-Association/Robot-Terms-and-Definitions/productid/2953. Las interacciones humano-robot exigen definir nuevas reglas de conducta y también reglas técnicas que doten de seguridad dicha coexistencia. Hay que tener cierta precaución en los términos que se usan, ya que los especialistas en robótica hablan a veces de LAWS, pero no se refieren a leyes, sino a la abreviatura en inglés Lethal Autonomous Weapon Systems, que es un campo en el que surgen problemas legales muy importantes. Véase Gutiérrez Espada, C. y Cervell Hortal, M. J. (2013): $27-57$.
} 
concepto occidental, aunque existe una idea común acerca de lo que es un robot industrial, no es fácil ponerse de acuerdo a la hora de establecer una definición formal. Además, la evolución de la robótica ha ido obligando a diferentes actualizaciones de su definición. Ya hemos mencionado antes a la RIA, según la cual un robot industrial es un manipulador multifuncional reprogramable, capaz de mover materias, piezas, herramientas, o dispositivos especiales, según trayectorias variables, programadas para realizar tareas diversas ${ }^{29}$.

Esta definición es adoptada por la Organización Internacional de Estándares (ISO) a la que nos hemos referido al citar el trabajo de Mercader Uguina. Una definición más completa es la establecida por la Asociación Francesa de Normalización (AFNOR $)^{30}$, que define primero el manipulador y después al robot:

- Manipulador: mecanismo formado generalmente por elementos en serie, articulados entre sí, destinado al agarre y desplazamiento de objetos. Es multifuncional y puede ser gobernado directamente por un operador humano o mediante dispositivo lógico.

- Robot: manipulador automático servo-controlado, reprogramable, polivalente, capaz de posicionar y orientar piezas, útiles o dispositivos especiales, siguiendo trayectorias variables reprogramables, para la ejecución de tareas variadas. Normalmente tiene la forma de uno o varios brazos terminados en una muñeca. Su unidad de control incluye un dispositivo de memoria y ocasionalmente de percepción del entorno. Normalmente su uso es el de realizar una tarea de manera cíclica, pudiéndose adaptar a otra sin cambios permanentes en su material.

Por último, la Federación Internacional de Robótica (IFR) ${ }^{31}$ distingue entre robot industrial de manipulación y otros robots: Por robot industrial de manipulación se entiende una máquina de manipulación automática, reprogramable y multifuncional con tres o más ejes que pueden posicionar y orientar materias, piezas, herramientas o dispositivos especiales para la ejecución de trabajos diversos en las diferentes etapas de la producción industrial, ya sea en una posición fija o en movimiento. En esta definición se debe entender que la reprogramabilidad y la multifunción se consiguen sin modificaciones físicas del robot.

Común en todas las definiciones anteriores es la aceptación del robot industrial como un brazo mecánico con capacidad de manipulación y que incorpora un control más o menos complejo. Un sistema robotizado, en cambio, es un concepto más amplio. Engloba todos aquellos dispositivos que realizan tareas de forma automática en sustitución de un ser humano y que pueden incorporar o no a uno o varios robots, siendo esto último lo más frecuente.

Si pasamos al plano jurídico, el problema se hace más complejo y, a estos efectos, es razonable y fundada la opinión de Palmerini ${ }^{32}$ quién señala que "todo fenómeno nuevo, especialmente cuando está ligado al desarrollo científico y tecnológico, no requiere necesariamente de la introducción de normas nuevas, ante la posibilidad de ser regulado por el sistema existente, capaz de adquirir una gran elasticidad". En el mismo sentido se expresa Lacruz Mantecón ${ }^{33}$ cuando pone como ejemplo el caso del llamado Derecho de internet, donde finalmente resultó que los problemas suscitados por esta nueva tecnología recibieron un tratamiento interdisciplinar, desde diferentes especialidades jurídicas poco o nada próximas, y no fue necesario crear un nuevo cuerpo de normas. Así, siguiendo a estos autores, no sería necesario elaborar un cuerpo de normas específicas para los robots, pues podrían adaptarse las normas ya existentes, pero el problema está en que estas normas preexistentes se deberían adaptar a ese nuevo fenómeno evitando provocar fricciones en el sistema. Por otra parte, en nuestra opinión, también hay que tener en cuenta que es el propio mercado el que demanda una cierta regulación de los robots, ya que su entrada en el mundo laboral, social, etc. tiene un impacto importante, no solo económico, y que esto afectará a las líneas de investigación y a las inversiones que se consideren adecuadas en el futuro.

\subsection{Los esfuerzos de las instituciones de la UE}

Aunque hablaremos más tarde de la opinión, muy crítica, de la doctrina española sobre la siguiente iniciativa, hay que decir que en nuestro entorno destacan los esfuerzos de la Unión Europea, que comenzó a ocuparse del tema hace ya algunos años. Son específicamente dos instituciones las que más se han preocupado y ocupado del impacto de los robots, la Comisión y el Parlamento Europeo. Efectivamente, el primer paso lo dio la Comisión de Asuntos jurídicos del Parlamento Europeo, que presenta el 31 de mayo de 2016 un proyecto de informe del que es ponente la socialista Mady Delvaux, que será el germen de la famosa Resolución del Parlamento Europeo, presentada el 16 de febrero de 2017 , con una serie de recomendaciones destinadas a la Comisión sobre normas de Derecho civil sobre robótica ${ }^{34 ;}$ en ella, la institución europea señalaba que "es necesario crear una definición generalmente aceptada de robot y de inteligencia artificial que sea flexible y no lastre la innovación” por lo que pide a la Comisión que proponga definiciones europeas

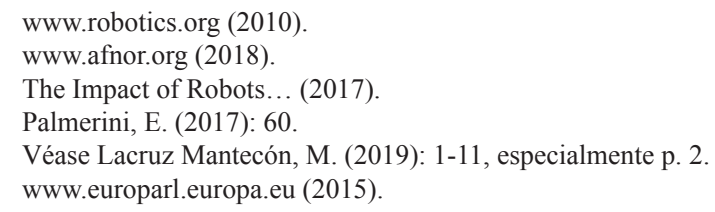


comunes $^{35}$ de sistema ciberfísico, sistema autónomo, robot autónomo inteligente y sus distintas subcategorías, tomando en consideración algunas características que considera propias de un robot inteligente, como son:

- capacidad de adquirir autonomía mediante sensores y/o mediante el intercambio de datos con su entorno (interconectividad) y el intercambio y análisis de dichos datos;

- $\quad$ capacidad de autoaprendizaje a partir de la experiencia y la interacción (criterio facultativo);

- $\quad$ un soporte físico mínimo;

- capacidad de adaptar su comportamiento y acciones al entorno;

- $\quad$ inexistencia de vida en sentido biológico

De estos rasgos podemos deducir que algunos ingenios que a primera vista podríamos considerar robots, no lo serían, por ejemplo, los ciborgs en la definición japonesa, pues son personas "mejoradas" o los programas de ordenador que realizan determinadas tareas útiles para el ser humano pero que no tienen soporte físico.

A los pocos meses, la Comisión Europea presenta una Comunicación en la que se hace referencia directa a la

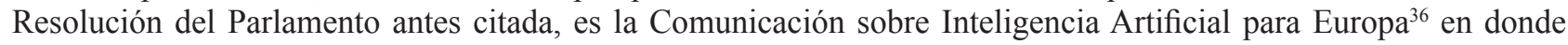
indirectamente, sin definir el concepto de robot, se hace referencia a ellos separándolos de la IA:

"Los sistemas basados en la IA pueden consistir simplemente en un programa informático (por ejemplo, asistentes de voz, programas de análisis de imágenes, motores de búsqueda, sistemas de reconocimiento facial y de voz), pero la IA también puede estar incorporada en dispositivos de hardware (p. ej. robots avanzados, automóviles autónomos, drones o aplicaciones del internet de las cosas)".

A riesgo de ser considerada una afirmación de Perogrullo, en nuestra opinión, está claro que robot e inteligencia artificial no son la misma cosa, pero sí es evidente que solo los robots con inteligencia artificial pueden dar lugar a problemas con los que, en principio, las normas jurídicas actuales no han contado. Esto es porque los robots sin inteligencia artificial pueden ser considerados cosas, sin más, en el ámbito del Derecho y en cambio, sobre los que tienen inteligencia artificial hay opiniones divergentes al respecto entre los especialistas.

Tras las propuestas de Parlamento y Comisión, el Comité Económico y Social Europeo presentó su Dictamen sobre la Inteligencia artificial: las consecuencias de la inteligencia artificial para el mercado único (digital), la producción, el consumo, el empleo y la sociedad ${ }^{37}$, en el que refiriéndose a la Resolución del Parlamento se muestra categórico en relación a varias de las afirmaciones allí contenidas:

- $\quad$ se opone a cualquier tipo de estatuto jurídico para los robots o sistemas de IA por el riesgo moral inaceptable que ello conlleva.

- $\quad$ se opone a que se compare la posible responsabilidad de los robots con la responsabilidad limitada de las sociedades, puesto que el responsable en última instancia es siempre una persona física en este último caso, cosa que no podría darse, en principio, en el caso de los robots

- $\quad$ indica que para abordar adecuadamente la legislación y la normativa en materia de IA se requiere entender muy bien que es lo que puede conseguir la IA y lo que no a corto, medio y largo plazo.

- $\quad$ es importante examinar la necesidad de una reglamentación mundial,

- como la IA no conoce fronteras, la normativa regional será insuficiente e incluso de efectos no deseados, por lo que recomienda que la UE adopte un papel de liderazgo estableciendo marcos universales y uniformes para la IA, y que fomente este proceso a nivel mundial.

Por su parte, al año siguiente la Comisión avanza presentando un documento preparatorio para iniciar un procedimiento legislativo, una Propuesta de Reglamento por el que se establece el programa Europa Digital para el período 2021-202738 cuyo fin es establecer los objetivos del programa, el presupuesto para el período 2021-2027, las formas de financiación de la Unión Europea y las normas para la concesión de dicha financiación. Entre sus definiciones no aparece el término robot, aunque sí integra entre sus prioridades la robótica y hace referencia a la inteligencia artificial. Hay que decir que, a los efectos de definir el concepto de robot, no añade nada a lo presentado hasta ahora por las instituciones de la UE.

\footnotetext{
Existen ya algunas definiciones de concepto conexos con el de robot presentadas por el PE, como la de persona electrónica: status legal potencial de robots autónomos susceptibles de ser sujetos de derechos y obligaciones específicos, incluyendo los de reparar cualquier daño que puedan causar. Estarían dotados de personalidad jurídica electrónica algunos robots capaces de tomar decisiones autónomas o interactuar de manera independiente con terceros. Veáse Comité de Asuntos Legales del Parlamento Europeo (2016). También el concepto de ciborg: ser formado por materia viva y dispositivos electrónicos (es definición de la RAE) aunque en Japón el concepto difiere algo, pues allí el ciborg es la persona que ha incorporado a su anatomía dispositivos que le permiten disponer de capacidades superiores a las naturales.

36 Comunicación de la Comisión al Parlamento Europeo, al Consejo Europeo, al Consejo, al Comité Económico y Social Europeo y al Comité de las Regiones titulada Inteligencia Artificial para Europa, Bruselas, 25.4.2018 COM (2018) 237 final.

37 Pleno de 31 de mayo y 1 de junio de 2017, (DO C 288, de 1 de agosto de 2017), véase especialmente párrafo 3.33 del Dictamen. Esta divergencia de opiniones es notada por Pérez Vaquero, C. (2019).

38 Bruselas, 6.6.2018 COM(2018)... (2018).
} 
Sin embargo, la Comisión ha realizado ya una construcción institucional en estas materias creando la unidad A.1, específicamente dedicada a Robótica e Inteligencia Artificial ${ }^{39}$ cuya misión es el desarrollo de una industria competitiva en este ámbito en Europa, que incluya robots industriales y de servicio, así como el creciente campo de sistemas autónomos que abarcan desde drones y vehículos sin conductor hasta visión cognitiva e informática. Pero esta unidad también se ocupa de las cuestiones éticas y legales relacionadas con los robots y los sistemas autónomos, como la responsabilidad y la seguridad, así como los aspectos relacionados con el impacto de la automatización y la robótica en el entorno laboral. También, tras un proceso de selección abierto, la Comisión nombró, en junio de 2018, a 52 especialistas para formar un Grupo de expertos de alto nivel sobre inteligencia artificial, compuesto por representantes de la academia, la sociedad civil y la industria ${ }^{40}$.

Este Grupo, el 8 de abril de 2019, presentó un primer borrador de unas Pautas éticas para el desarrollo y uso de la Inteligencia Artificial $^{41}$, documento que tiene su base en un primer proyecto de directrices éticas publicado por el mismo Grupo en diciembre de 2018, al que siguieron una consulta con las partes interesadas y reuniones con representantes de los Estados miembros para recabar sus opiniones. Estas pautas son una de las dos cuestiones en las que el Grupo ha trabajado en el primer año desde su creación, pues también ha presentado unas Recomendaciones de política e inversión ${ }^{42}$ que pretendían ayudar a la Comisión y a los Estados miembros a actualizar a finales de 2019 un plan coordinado conjunto sobre IA que se había presentado en diciembre de $2018^{43}$.

El Parlamento Europeo presenta, por su parte, una Resolución de 12 de febrero de 2019, sobre una política industrial global europea en materia de inteligencia artificial y robótica ${ }^{44}$ que no incluye una nueva definición de robot, pero sí recomienda la creación de normas comunes en materia de robots e IA que respeten los valores fundamentales de la Unión y habla de un concepto que puede resultarnos interesante a los efectos de este estudio: habla de la importancia del principio de reconocimiento mutuo en el uso transfronterizo de "bienes inteligentes", incluidos los robots y los sistemas robóticos.

Por otra parte, este mismo documento, en la línea de lo que ya hemos afirmado sobre la necesidad, o no, de nuevas regulaciones específicas sobre robots, indica (en materia de propiedad intelectual, pero podría extenderse a otros ámbitos) que "no hay ninguna disposición jurídica que se aplique específicamente a la robótica, pero que las doctrinas y los regímenes jurídicos actuales pueden aplicarse fácilmente a esta, aunque algunos aspectos requieran especial consideración".

Parece que la propuesta presentada por el Parlamento Europeo ha perdido gran parte de la fuerza que tuvo cuando se adoptó, e incluso ese impulso que se daba a la Comisión para que presentase una propuesta de Directiva recogiendo el contenido de la Resolución tampoco ha continuado y no se prevé la presentación de ninguna iniciativa legislativa en este sentido ${ }^{45}$.

\subsection{La opinión de los expertos en España. Críticas a la construcción europea.}

Dado que en un trabajo de estas características es imposible abordar una revisión completa de las opiniones de expertos de todos los Estados miembros de la UE, nos vamos a circunscribir a un somero análisis de las opiniones de la doctrina española sobre esta cuestión.

En España nos encontramos ya con una serie de estudios en el ámbito del Derecho, publicados en fechas muy recientes la mayor parte de ellos, y normalmente en forma de trabajos colectivos, como los coordinados por el catedrático de Derecho del trabajo, Jesús Mercader Uguina ${ }^{46}$, los trabajos de Carlos Rogel Vide ${ }^{47}$ o Javier Ercilla García $^{48}$ en el ámbito del Derecho civil, los de Rafael de Asís ${ }^{49}$ en el ámbito de los derechos humanos o los de Moisés Barrio Andrés ${ }^{50}$ de carácter transversal.

ec.europa.eu $(\mathrm{s} / \mathrm{f})$.

40 De los cincuenta dos expertos europeos, solo dos eran de nacionalidad española: Lorena Jaume-Palasí y Cristina San José.

${ }^{41}$ Se enumeran los 7 requisitos clave que los sistemas de inteligencia artificial deben cumplir para ser confiables. Estos requisitos pasarán por un proceso piloto que se espera concluya con la presentación de un documento revisado a principios de 2020. Las pautas se pueden consultar en todos los idiomas oficiales de la UE, véase ec.europa.eu (2018).

42 Véase ec.europa.eu (2019).

43 Este plan fue presentado en 2018 por la Comisión (véase Comunicación de la Comisión al Parlamento Europeo, al Consejo Europeo, al Consejo, al Comité Económico y Social Europeo y al Comité de las Regiones, Plan coordinado sobre la inteligencia artificial, Bruselas, 7.12.2018 COM (2018) 795 final) y debería haberse publicado una actualización a finales de 2019, pero por el momento esto no ha sucedido.

44 Texto aprobado P8_TA (2019) 0081. www.europarl.europa.eu (2019).

45 A pesar de que algunos han tratado esta cuestión como posible, por ejemplo, véase la noticia "La Comisión Europea no tiene intención de crear estatus legal para los robots". La Vanguardia, 23 de abril de 2018, en donde se indica la existencia de una carta abierta a la Comisión, proveniente de la iniciativa "Robotics, Open Letter" en la que sus firmantes anunciaban que la Comisión sí está preparando "una nueva directiva sobre robótica e inteligencia artificial que otorgará personalidad jurídica a las máquinas". Los últimos desarrollos que se tienen en cuenta como avances en relación con la Resolución del Parlamento Europeo son los presentados por el Grupo de Alto Nivel de la Comisión (Merino, M. (2028)), o Los robots y la inteligencia artificial impulsan sus propias leyes, 11 de julio de 2019. Expansión.

46 Véase Mercader Uguina, J. (2017).

47 Rogel Vide, C. et al. (2018).

48 Ercilla García, J. (2018).

49 De Asis, R. (2015).

50 Barrio Andrés, M. et al. (2018). 
En los documentos presentados tanto por la Comisión como por el Parlamento Europeo que acabamos de revisar, puede parecer que hay una tendencia a otorgar una cierta personalidad jurídica a los robots, al menos así parece deducirse de la Resolución del Parlamento cuando pide específicamente a la Comisión que presente una propuesta de Directiva que de un marco legal claro y que "se estudie la posibilidad de crear una personalidad jurídica específica para los robots" 51 . Esta propuesta ha sido analizada con cuidado por algunos expertos, por ejemplo, Aransay Alejandre ${ }^{52}$ que, con mucha sensatez, dice que la eventual creación de una personalidad jurídica para los robots, si se avanzara en esta línea, no se debería limitar a otorgar una serie de derechos y obligaciones para los robots sino que sería necesario plantear si esta capacidad sería plena o limitada. La autora piensa que eso obligaría al legislador a adoptar figuras propias del Derecho de familia. En todo caso, dice, carecer de una definición de robot en el plano jurídico nos impide todavía dar estos pasos con garantías de éxito. Más aún, añaden otros autores, como Rosales De Salamanca ${ }^{53}$, que el problema no es tanto atribuir o no personalidad jurídica a los robots sino dotarles de capacidad de obrar, determinar qué acciones pueden realizar como sujetos de Derecho. Y continúa diciendo que, si reconocemos la personalidad jurídica de un robot, lo difícil será fijar el límite entre la personalidad jurídica y la propiedad del objeto, pues las relaciones jurídicas siempre se entablan entre personas y no entre personas y cosas o entre cosas, y porque hay tantos tipos de robots posibles, que en ocasiones habrá que acudir a la personalidad jurídica y en otras a la figura de la propiedad. Otros especialistas proponen analizar quién es titular de los robots y quién puede usarlos ${ }^{54}$ pues, como sabemos, el aprendizaje de ciertos robots es progresivo y, en consecuencia, sus actos pueden ser resultado del programa que usan o del aprendizaje y del uso que de dicho programa haya realizado su dueño o persona encargada de la formación de la máquina en cuestión.

Rogel Vide ${ }^{55}$ tiene una opinión muy crítica sobre algunas de las consideraciones que se incluyen en los trabajos del Parlamento Europeo y así, en respuesta a la posible consideración de los robots como personas, personas electrónicas o sujetos de derechos, indica que:

“(...) los robots son cosas, todo lo singulares que se quiera, mas no pueden ser personas, sujetos de derechos, y sí solo objetos de los derechos dichos, singularmente el de propiedad y cualesquiera otros reales. Son cosas especiales, ciertamente, capaces de realizar operaciones complejas y de sustituir a las personas en muchas de sus tareas, incluso en épocas -como la nuestra- de crisis y paro".

En el mismo sentido, Lacruz Mantecón afirma "Las creaciones tecnológicas son eso, creaciones humanas" 56 , Jaume Palasín ${ }^{57}$ también sigue este mismo razonamiento cuando afirma que la IA es reflejo de los seres humanos, la hemos creado nosotros y, por ello, es proyección y no superación del ser humano. Y también fuera de España es una opinión extendida ${ }^{58}$.

Lo cierto es que la escasa doctrina jurídica española que por ahora ha estudiado el tema se ha caracterizado por centrarse en uno o varios aspectos concretos del impacto de la robótica en la sociedad (ya sea en el ámbito del Derecho del trabajo, ya en las cuestiones éticas, ya en los problemas jurídicos que surgen del uso de armas autónomas en la defensa...) pero son muy pocos los autores que han intentado dar una definición de robot válida de manera general en todos los ámbitos del Derecho. Además, se da la circunstancia de que, en esos pocos intentos de definición, los autores han tomado como base la Resolución del Parlamento Europeo de 2017, a la que también hemos hecho referencia en este trabajo, Resolución que, con el paso de muy pocos años, parece que ha quedado relegada u olvidada, ya que las propias instituciones europeas que la impulsaron no han seguido las recomendaciones que contiene y no parece probable que su principal objetivo (servir de base a una propuesta de Directiva que regule a los robots) vaya a verse cumplido, al menos en un futuro cercano.

\section{Conclusiones}

Podemos concluir este somero repaso con la extracción de varias ideas centrales que contribuyan a aclarar, siquiera sea solo en cierta medida, los conceptos tratados:

\footnotetext{
51 Concretamente la Resolución del Parlamento Europeo pide a la Comisión en su párrafo 59.f) “crear a largo plazo una personalidad jurídica específica para los robots, de forma que como mínimo los robots autónomos más complejos puedan ser considerados personas electrónicas responsables de reparar los daños que puedan causar, y posiblemente aplicar la personalidad electrónica a aquellos supuestos en los que los robots tomen decisiones autónomas inteligentes o interactúen con terceros de forma independiente".

52 Véase Aransay Alejandre, A.M. (2018): 87-106, especialmente 101-102.

53 El autor puntualiza que la Resolución del PE se refiere a la inteligencia artificial fuerte, que no existe todavía, de hecho, hay problemas técnicos reales para lograr esa inteligencia artificial fuerte y mientras los ordenadores dependan del silicio, va a ser más que difícil lograr la capacidad de computación suficiente Rosales De Salamanca, F. (2016).

54 Sánchez Del Campo Redonet, A. (2017).

55 Rogel Vide, C. (2018).

56 Lacruz Mantecón, M. (2019): 1-6.

57 Así lo afirmaba en la Jornada organizada por Fundación para la Investigación en Derecho y Empresa (FIDE) el 21 de enero de 2020 en Madrid en su comunicación "La IA y la democracia en el siglo XXI. Iniciativas y retos".

58 Véase, por todos, Leroux, Ch. y otros, (2012): 11, que afirma con toda claridad: "Robots are artefacts, instruments in the hands of manufacturer, programmer, owner and user".
} 
Los intentos de definición del término 'robot' en Japón y en el entorno europeo se hallan en una situación bastante similar, pues en ambas áreas geográficas se parte de una serie de conceptos que provienen, sobre todo, del ámbito de la ingeniería (normas JIS e ISO), pero en el estadio en que nos encontramos, deberíamos intentar adoptar un concepto amplio de robot, omnicomprensivo de las diferentes innovaciones tecnológicas. Lo cierto es que hay muchos tipos de robots y muchos ámbitos en los que resultan útiles, y todos ellos deberían ser integrados dentro de una misma categoría.

Cuestión diferente es su consideración jurídica, a este respecto nos parece adecuada la solución propuesta por algunos juristas que sugieren no crear un específico "Derecho de los robots", no sobrelegislar y, cuando sea necesario establecer regulaciones específicas, que cada una contemple su propia definición de robot, salvo que opte por la posibilidad de remitir a otra preexistente, si resulta adecuada a su ámbito de aplicación y a sus fines.

Respecto a las características que debe reunir un ingenio para ser calificado como robot, en la línea que ya hemos referido, nos inclinamos por pensar que debe considerarse tal a un sistema capaz de realizar tareas al servicio del ser humano de manera altamente automática, captando información del entorno, analizándola y respondiendo de modo autónomo en función de ella. Sin duda la clave de la cuestión radica en deslindar la actuación meramente automática de la altamente automática, pues ello permite excluir de la definición a ingenios que resultan demasiado habituales y simples en nuestros días, como las células fotoeléctricas que encienden y apagan las luces o las escaleras mecánicas que se accionan con la llegada de un usuario, aparatos sencillos que, de otro modo, encajarían en la definición antedicha.

Del mismo modo, respecto a su consideración jurídica, habrá ingenios y aparatos que deban ser considerados meras máquinas y otros, auténticos robots. Además, los que incluyan entre sus características el uso de IA, deberán ser objeto de una seria reflexión que tenga en cuenta el momento de avance tecnológico en el que nos encontremos y la capacidad de interrelación de esos robots con el ser humano, para determinar si las normas jurídicas deben considerarlos sujetos de derecho y, en consecuencia, atribuirles un cierto grado de responsabilidad, al modo de la que incumbe a las personas jurídicas (responsabilidad de persona electrónica) o tratarlos como creaciones potencialmente generadoras de responsabilidad para sus fabricantes, programadores, manipuladores, propietarios, mantenedores, etc.

En el momento actual, considero que todos ellos, incluso los dotados de IA, no deben ser considerados sujetos de derecho. En esto me uno a la opinión mayoritaria de la doctrina española. Efectivamente, gran parte de los civilistas y también juristas de otros ámbitos, opinan que los robots no pueden ser considerados personas a efectos jurídicos, son objeto de derecho, pero no sujetos. También puede afirmarse que la mayoría aboga por no crear una nueva rama del Derecho que podría denominarse Derecho de los robots, como ya hemos avanzado, salvo quizás Barrio Andrés que ha dirigido varias obras colectivas con denominaciones en este sentido.

En una definición que aspire a ser omnicomprensiva, como la que propugnamos, la necesidad de un soporte físico resulta una exigencia fundamental, pero no sería necesario que ese soporte físico tuviera apariencia humana, aunque lo cierto es que el público en general asume que este tipo de ingenios humanoides son robots. Para el ámbito del Derecho, lo que constituye una diferencia fundamental es la capacidad del robot de actuar más allá de unas instrucciones dadas, usando el aprendizaje progresivo, y no tanto el ámbito industrial, sanitario u otros en que el robot vaya a ser utilizado. Finalmente, los intentos de las instituciones de la Unión Europea por definir el concepto de robot, aunque válidos como una primera aproximación, no parece que vayan a tener un gran recorrido, pues la principal contribución ha sido la del Parlamento Europeo, que proponía la elaboración de una norma vinculante, una Directiva, y ni siquiera se han iniciado todavía los trabajos de los legisladores comunitarios en relación con este tema.

Estas conclusiones deben ser solo una primera aproximación, sin perder de vista que estamos analizando un fenómeno emergente en el ámbito jurídico, no en el tecnológico. Sin embargo, estos primeros pasos van a ser determinantes para analizar la repercusión de los robots en nuestra disciplina y hay que darlos con sumo cuidado, legislar de modo irreflexivo o dejándose llevar por una u otra corriente puede tener graves consecuencias, no solo para la economía, el mundo laboral o la política, sino para el avance del ser humano en general.

\section{Bibliografia}

Anzai, Yuichiro et al. (2004): Creación de la Robótica, Cursos Iwanami, Robótica 1 (ロボット学創成 ロボットガク ソウセイ). Tokio: Iwanami Shoten.

Aransay Alejandre, Ana $\mathrm{M}^{\mathrm{a}}$ (2018): “Antecedentes y propuestas para la regulación jurídica de los robots”. En: Moisés Barrio Andrés (Dir.) Derecho de los robots. Madrid: La Ley, Wolters Kluver España, pp. 87-106.

Asami, Shuji, (1994): “Robots in Japan: Present and Future”. En: IEEE Robotics \& Automation Magazine, junio, Bruselas, pp. 22-26.

Barrio Andrés, Mario y otros (2018): Derecho de los robots. Madrid: La Ley.

Bertolini, Andrea / Palmerini, Erica (2014): "Regulating Robotics: A Challenge for Europe". En: European Parliament, Policy Department C: Citizens' Rights and Constitutional Affair, Workshop for the Juri Committee. Bruselas, pp. 167202. https://www.europarl.europa.eu/RegData/etudes/IDAN/2014/509987/IPOL_IDA(2014)509987(ANN01)_EN.pdf.

Bruselas, 6.6.2018 COM(2018) 434 final. 2018/0227 (COD) (2018). En: Eur-Lex. Access to European Union Law. https:// eur-lex.europa.eu/legal-content/ES/ALL/?uri=CELEX\%3A52018PC0434 [Consulta: 14/01/2020] 
Cisneros Cristóbal, Paula (2019): "El Acuerdo de Asociación económica entre la UE y Japón: negociación, contenido y análisis de elementos clave". En: Carmen Tirado Robles / Francisco Barberán (Coord.) Derecho y Relaciones internacionales en Japón desde el Tratado de amistad, comercio y navegación de 1868. Zaragoza: Prensas de la Universidad de Zaragoza, pp. 173-209.

Comité de Asuntos Legales del Parlamento Europeo (2016): Informe preliminar de 31 de mayo de 2016, acerca de legislación civil para regular la robótica. Parlamento Europeo. https://www.europarl.europa.eu/committees/es/juri/ robotics.html?tab=Antecedentes . [Consulta: 14/01/2020]

Conde Canaviri, Martha (2008): “Generaciones de la robótica”. En: Revistas Bolivianas, No. 1, La Paz, pp. 126-130 [http://www.revistasbolivianas.org.bo/pdf/rits/n1/n1a32.pdf]

De Asis, Rafael (2015): Una mirada a la robótica desde los derechos humanos. Colección: Cuadernos "Bartolomé de las Casas". Madrid: Editorial Dykinson.

De La Parra Trujillo, Eduardo (2017), “Aplicación de normas jurídicas y antinomias normativas en Yo robot de Isaac Asimov”. En: Oscar Enrique Torres (Coord.) Derecho \& Literatura. El derecho en la literatura. México: Editorial Libitum, pp. 165-188.

ec.europa.eu (2018): Ethics Guidelines for Trustworthy AI. European Commission. https://ec.europa.eu/futurium/en/aialliance-consultation/guidelines\#Top. [Consulta: 14/01/2020]

ec.europa.eu (2019): Policy and investment recommendations for trustworthy Artificial Intelligence. European Commission. https://ec.europa.eu/digital-single-market/en/news/policy-and-investment-recommendations-trustworthy-artificialintelligence. [Consulta: 14/01/2020]

ec.europa.eu (s/f): Robotics and Artificial Intelligence (Unit A.1). European Commission. https://ec.europa.eu/digitalsingle-market/en/content/robotics-and-artificial-intelligence-unit-a1 [Consulta: 14/01/2020]

Ercilla García, Javier (2018). Derecho Civil y Robótica. Robots Inteligentes, Personalidad Jurídica, Responsabilidad Civil y Regulación. Cizur Menor: Thomson Reuters Aranzadi.

Esteve, Ferrán, (2015): “La hipótesis del «valle inquietante» en los robots”. En: CCCBLAB, Investigación e innovación en cultura, Dossier Inteligencia artificial, 11 de noviembre de 2015. http://lab.cccb.org/es/la-hipotesis-del-valleinquietante-en-los-robots/.

García-Prieto Cuesta, Juan (2018): “¿Qué es un robot?”, En: Moisés Barrio Andrés (ed.). Derecho de los robots. Madrid: La Ley, Wolters Kluwer España, pp. 27-60.

Gascón Marcén, Ana (2020): "Society 5.0: EU-Japanese cooperation and the opportunities and challenges posed by the data economy". En: Análisis Real Instituto Elcano, documento No. 11/2020, Madrid, http://www.realinstitutoelcano. org/wps/portal/rielcano_en/contenido?WCM_GLOBAL_CONTEXT=/elcano/elcano_in/zonas_in/ari11-2020gascon-society-5-0-eu-japanese-cooperation-and-opportunities-and-challenges-posed-by-data-economy.

Gutiérrez Espada, Cesáreo / Cervell Hortal, Ma José (2013): "Sistemas de armas autónomas, drones y Derecho internacional”. En: Revista del Instituto Español de Estudios Estratégicos, No. 2, Madrid, pp. 27-57.

Hirano, Shin (2017): Derecho de los robots (『ロボット法』-AIとヒトの共生にむけて). Tokio: Koubundo publishers.

Hornyak, Timothy N. (2006): Loving the Machine: The Art and Science of Japanese Robots, New York: Kodansha International.

Introducción al Acuerdo de Asociación económica UE-Japón, Cooperación en materia de reglamentación y medidas no arancelarias, Comisión Europea (2017). Trade Websites. https://trade.ec.europa.eu/doclib/docs/2017/july/ tradoc_155944.pdf [Consulta: 14/01/2020]

j-net21.smrj.go.jp (2020): ¿Cuáles son los principios básicos para introducir un robot en el lugar de trabajo? J-Net21. https://j-net21.smrj.go.jp/qa/productivity/Q1077.html [Consulta: 14/01/2020]

JETRO (Japan External Trade Organization) (2004): Marcado y etiquetado, LEGISCOMEX. https://www.legiscomex. com/comunicaciones/n/nov-17-04/marcado-etiquetado-japon.pdf [Consulta: 14/01/2020]

kikakurui.com (2012). Kikakuri. https://kikakurui.com/b0/B0134-2015-01.html [Consulta: 14/01/2020]

Lacruz Mantecón, Miguel Luis (2019): “Cibernética y Derecho Europeo: ¿una inteligencia robótica?”. En: Diario La Ley, No. 9376, Madrid, pp. 1-11.

Lacruz Mantecón, Miguel Luis (2019): “Potencialidades de los robots y capacidades de las personas”. En: Revista General de Legislación y jurisprudencia, No. 1, Madrid, pp. 85-129.

Lacruz Mantecón, Miguel Luis (2019): "Sé que estás mintiendo, o los límites de la Inteligencia artificial (1)". En: Revista de Derecho del Mercado de Valores, No. 25, Madrid, pp. 1-6.

Leroux, Chistophe et al. (2012): Suggestion for a green paper on legal issues in robotics. En: The European Robotics Coordination Action, Grant Agreement Number: 248552, Contribution to Deliverable D3.2.1 on ELS issues in robotics. https://www.unipv-lawtech.eu/files/euRobotics-legal-issues-in-robotics-DRAFT_6j6ryjyp.pdf.

Martínez De Pisón, José Ma (2017): “Yo, Robot: de la biología a la singularidad. ¿Nuevas preguntas para la Filosofía del Derecho?”. En: Revista electrónica del Departamento de Derecho de la Universidad de La Rioja, No. 15, diciembre, Logroño, pp. 57-73, https://publicaciones.unirioja.es/ojs/index.php/redur/article/view/4161/3411.

Mercader Uguina, Jesús (2017): El futuro del trabajo en la era de la digitalización y la robótica. Valencia: Tirant lo Blanc. Mercader Uguina, Jesús (2018): "Robótica y riesgos laborales". En: Archivos de Prevención de Riesgos Laborales, Vol. 21, No. 3, julio-septiembre, Barcelona, http://scielo.isciii.es/scielo.php?script=sci_arttext\&pid=S157825492018000300001\#aff1 
Merino, Marcos (20 de diciembre 2018): LaUE presenta sus directrices para el desarrolloético de la inteligencia artificial.Xataka. https:// www.xataka.com/robotica-e-ia/ue-presenta-sus-directrices-para-desarrollo-etico-inteligencia-artificial. [Consulta: 14/01/2020]

Ministry of Economy, Trade and Industry of Japan (10 de febrero 2015): New Robot Strategy, Japan's Robot StrategyVision, Strategy, Action Plan. En: Ministry of Economy, Trade and Industry. https://www.meti.go.jp/english/ press/2015/pdf/0123_01b.pdf [Consulta: 14/01/2020]

Ministry of Economy, Trade and Industry of Japan (2006): Informe del grupo de estudio sobre políticas de robots (2006 年5月（経済産業省の）ロボット政策研究会報告書). En: Japan Robot Association. https://www.jara.jp/various/ report/img/robot-houkokusho-set.pdf [Consulta: 14/01/2020]

Mori, Masahiro (12 de junio 2012): “The Uncanny Valley: The Original Essay by Masahiro Mori”, traducción de Karl F. MacDorman \& Norri Kageki autorizada por el autor. En: IEEE Robotics \& Automation Magazine. https://spectrum. ieee.org/automaton/robotics/humanoids/the-uncanny-valley

Nanbu, Tomoko (2019): ロボット法（ロボットに関する法律問題 (Cuestiones legales sobre robots”). En: River-City Legal Profession Corporation, Ichikawa https://www.rclo.jp/general/report/cat03/21/ .

Ordinance on Industrial Safety and Health, based on the Industrial Safety and Health Act (Act No. 57 of 1972) and the Enforcement Order of Industrial Safety and Health Act (Cabinet Order No. 318 of 1972) (1972). En: Japanese Law Translation. http://www.japaneselawtranslation.go.jp/law/detail_main?re=01\&ia=03\&vm=02\&id=1984. [Consulta: 14/01/2020]

Palmerini, Erica (2017): "Robótica y derecho: sugerencias, confluencias, evoluciones en el marco de una investigación europea”. En: Revista de Derecho Privado, No. 32, Bogotá, pp. 53-97.

Pérez Vaquero, Carlos (2019): "La personalidad jurídica robótica, según la Unión Europea”. blog Iustopia. http:// archivodeinalbis.blogspot.com/2019/05/la-personalidad-juridica-robotica-segun.html .

Poole, Harry (1989): Fundamentals of robotics engineering. New York: Springer, Van Nostrand Reinhold.

Regulations for Enforcement of the Radio Act (Radio Regulatory Commission Rules No. 14 of November 30, 1950) (1950). En: Ministry of Internal Affairs and Communications, https://www.soumu.go.jp/main_sosiki/joho_tsusin/eng/ laws_dt02.html. [Consulta: 14/01/2020]

Rogel Vide, Carlos (2018): “Robots y personas”. En: Revista General de Legislación y Jurisprudencia, No. 1, 2018, Madrid, pp. 79-90.

Rogel Vide, Carlos y otros (2018): Los robots y el Derecho. Madrid: Reus Editorial.

Rosales De Salamanca, Francisco (12 de diciembre 2016): “¿Puede ser un robot sujeto de derecho?”. Notario Francisco Rosales. https://www.notariofranciscorosales.com/puede-robot-sujeto-derecho/ .

Sáiz Lorca, Daniel (2006): La literatura checa de ciencia ficción durante el período de entreguerras. Tesis doctoral dirigida por Alejandro Hermida de Blas, Universidad Complutense de Madrid. http://webs.ucm.es/BUCM/tesis//fll/ ucm-t\%2029008.pdf .

Sánchez Del Campo Redonet, Alejandro (2017): “Europa quiere regular a los robots”. En: Diario La Ley, 2253/2017, No. 4, Madrid.

Shishido, Joji, (2018), “Artificial intelligence and Society and Law”. En: 宍戸 常寿，大屋 雄裕，小塚荘一郎，佐 藤一郎，生貝 直人，市川 芳治 論究ジュリスト= Quarterly jurist (26), Tokio, pp. 114-130.

The Impact of Robots on Productivity, Employment and Jobs. Positioning Paper (2017). En: International Federation of Robotics. https://ifr.org/downloads/papers/IFR_The_Impact_of_Robots_on_Employment_Positioning_Paper_ updated_version_2018.pdf. [Consulta: 14/01/2020]

Venanzoni, Andrea (2019): "La valle del perturbante: il costituzionalismo alla prova delle intelligenze artificiali e della robotica". En: Politica del diritto, No. 2, Bolonia, pp. 237-280.

Wong, Simone (2017): “The Uncanny Valley Effect: Implications on Robotics and A.I. Development”. En: Introduction to Cognitive Science. MA Cognition and Communication, University of Copenhagen https://www.researchgate.net/ publication/328463012_The_Uncanny_Valley_Effect_Implications_on_Robotics_and_AI_Development .

www.afnor.org (2018): Legal Information. Afnor Group. https://www.afnor.org/en/legal-information/ . [Consulta: $14 / 01 / 2020]$

www.europarl.europa.eu (2015): Referencia A8-0005/2017, (2015/2103(INL)). Parlamento Europeo. http://www.europarl. europa.eu/doceo/document/TA-8-2017-0051_ES.html [Consulta: 14/01/2020]

www.europarl.europa.eu (2019): Una política industrial global europea en materia de inteligencia artificial y robótica. Parlamento Europeo. http://www.europarl.europa.eu/doceo/document/TA-8-2019-0081_ES.html . [Consulta: 14/01/2020]

www.jisc.go.jp/eng (2017): The Japanese Industrial Standards Committee. https://www.jisc.go.jp/eng/index.html www.robotics.org (19 de enero 2010): Robots Terms and Definitions. Robotics. https://www.robotics.org/product-catalogdetail.cfm/Robotic-Industries-Association/Robot-Terms-and-Definitions/productid/2953 [Consulta: 14/01/2020]

Yanaga, Masao (2014): “Standards and Statutes: 'Soft' Law and 'Hard' Law”. En: Sankai, Yoshiyuki / Suzuki, Kenji / Hasegawa, Yasuhisa (ed.). Cybernics. Fusion of human, machine and information systems. Tokyo: Springer Japan, pp. 315-333.

Zabala, Gonzalo (2007): Robótica. Guía teórica y práctica. Lomas de Zamora: Gradi. 